\title{
Die Logik der Praxis: 15 Jahre EQUAM Stiftung
}

\section{Marianne Jossen}

M.A., wissenschaftliche Mitarbeiterin EQUAM Stiftung

\author{
2014 wurde EQUAM 15 Jahre alt. Die Stiftung fördert und zertifiziert Qualität in \\ der ambulanten Medizin. Zum Jubiläum gibt es keine grosse Feier. Dafür eine von \\ Ärztinnen, Ärzten und einem Auditoren der Stiftung angeregte Reflexion darüber, \\ was erfolgreiche Qualitätsarbeit eigentlich ausmacht und wohin die Reise gehen \\ könnte.
}

Man soll der Praxis eine eigene Logik zuerkennen dafür setzte sich Pierre Bourdieu ein, nachdem er in den 5oer Jahren in Algerien die Kultur der Berber untersucht hatte [1]. Die Praxis sei ein situiertes Tun, so der Soziologe. Sie entziehe sich deshalb einer vollständigen Systematisierung. Klassifikationen, Regeln und Handlungsanweisungen würden mehr oder weniger flexibel eingesetzt und kontextspezifisch angepasst. Die Praxis habe eben ihre eigene Art und Weise, ihre eigene Logik im Umgang mit den systematischen Aspekten einer Kultur. In der Medizin und

\section{«Wir kontrollieren nicht.}

Wir schauen und beobachten.»

gerade in der Hausarztmedizin lassen sich durchaus ähnliche Spannungsverhältnisse beobachten. Wissenschaftliche Evidenz einerseits, individuelle Patienten andererseits. Medizinische Guidelines und organisatorische Standards hier, eine von Ereignissen getriebene Arbeit dort.

\section{Der Kern von Qualitätsbemühungen}

Die Arbeit an und in diesem Spannungsverhältnis als Herausforderung willkommen heissen und bewusst immer wieder Praxis und Systematik miteinander konfrontieren. Das ist ein Kern jeder Bemühung um Qualität. Zumindest lässt sich so die Erfahrung der EQUAM resümieren. Hans-Peter Wyss, seit zwölf Jahren für die Stiftung als Auditor tätig, meint dazu: «Die klassische Hausarztpraxis lebt von einer Spontaneität in der Gestaltung des Praxisalltags. Solche klassischen Praxen haben wir mit der Erkenntnis, dass Standardisierung Sicherheit bringt, zunächst überrascht.» Entscheidend ist die Überraschung. Qualitätsarbeit gelingt nur, wenn einerseits die Systematik Überraschungspotential birgt und wenn andererseits die tägliche Praxis sich irritierbar und offen zeigt für vielerlei Fragen. Dann kann eine erste Patientenbefragung eine «schrecklich schöne Erfahrung» werden, wie Michael Deppeler, EQUAM zertifizierter Hausarzt, zu Protokoll gibt.

\section{Gestern und heute}

Solche Erfahrungen verschafft EQUAM Grundversorgern und Spezialärztinnen seit nun 15 Jahren. Bei der Gründung ging es zunächst darum, zu zeigen, dass auch und gerade in einem Managed-Care-Kontext, damals als «Billig-Medizin» verschrien, qualitativ hochwertige Arbeit möglich ist. Man adaptierte und entwickelte Qualitätsprogramme für die Gesamtorganisation der Praxis, aber auch bezüglich der Behandlung chronischer Krankheiten oder für die Frage nach der Medikation. Heute ist EQUAM für alle diejenigen Arztpraxen eine Adresse, die Qualitätsmanagement als Führungs- und Reflexionsinstrument verstehen und nutzen wollen. Die Besuche der Auditoren werden zu einem Ereignis für die gesamte Organisation: «Unsere Mitarbeiterinnen waren stolz, dass ihre Arbeit, die sie das ganze Jahr leisten, geschätzt wurde», meint etwa Suzanne Aebi, Gynäkologin in einer Basler Doppelpraxis. Inzwischen sind rund 300 Stellen in der Deutschschweiz zertifiziert vom Institut für Arbeitsmedizin mit rund 90 Mitarbeiterinnen bis zur kleinen Bündner Landpraxis, wo Claudia Schertlin im Alleingang ihre Patienten betreut. Sie sagt: "Ich sehe die Zertifizierung als eine Ehre und als eine fortdauernde Verpflichtung, meine 


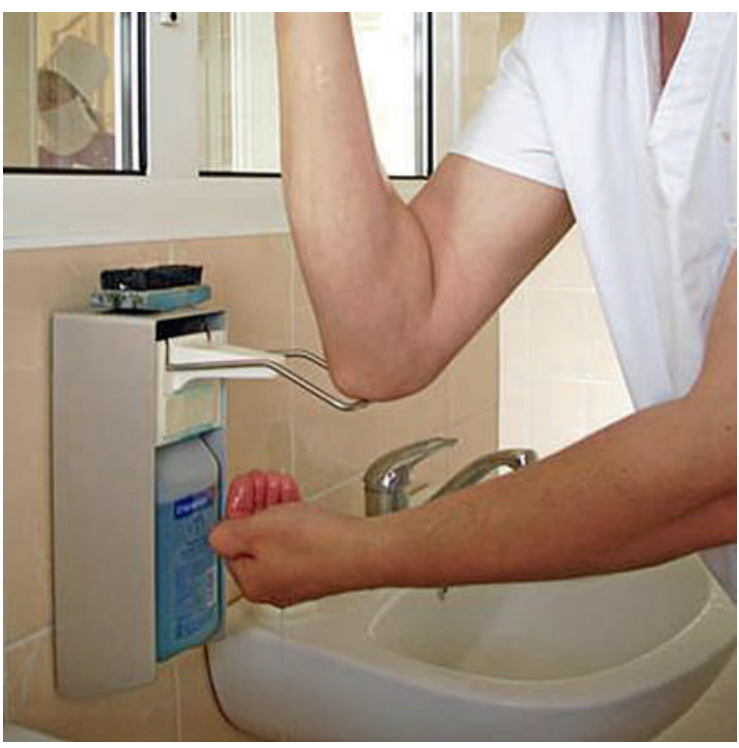

Qualitätsarbeit bedeutet auch Abläufe beobachten, optimieren und neue Routinen festlegen, zum Beispiel bei Hygienemassnahmen.

Praxis auf allen geprüften Terrains zeitgemäss und fortschrittlich weiterzuentwickeln.»

\section{Ein bisschen Zeit für neue Rituale}

Es ist klar, die Transparenz, die das EQUAM-Verfahren schafft, hat ihren Preis - und zwar nicht nur einen monetären, sondern insbesondere einen zeitlichen. Könnte man nicht einfach weiterarbeiten wie

\section{Personalfragen haben mit zunehmender Fluktuation und Grösse der Arztpraxen an Brisanz gewonnen.}

bisher? Muss man alles regeln? Nein, alles muss man nicht regeln. Aber gerade in Zeiten erhöhter Personal- und Patientenfluktuation macht es Sinn, wichtige Abläufe auf medizinische oder organisationswissenschaftliche Evidenz zu gründen und in Schriftform personenunabhängig zu machen. In die tägliche Routine, oder das, was Bourdieu «Praxis» nannte, eingelassen, werden solche Verfahren zu Ritualen, die jedoch - im Gegenteil zu ihren religiösen und vormodernen Pendants - hinterfragbar und anpassbar bleiben.

Im besten Fall entsteht dabei eine Verzahnung von Standard und Praxis. Als Beispiel nennt Hans-Peter Wyss die Hygiene: Hygienemassnahmen sind Elemente in dem Prozess, der die Patientin durch die Praxis leitet. Das Festlegen der Massnahmen strukturiert den Prozess. Gleichzeitig schützen Hygiene- massnahmen sowohl Patienten als auch Mitarbeitende. So entsteht «Ablaufsicherheit gleichzeitig mit Hygienesicherheit».

\section{Qualitätsarbeit als Spiegel der eigenen Praxis}

Wichtig ist im gesamten Verfahren, das die Arztpraxis in Begleitung der Stiftung durchläuft, die Haltung der Auditoren: «Wir kontrollieren nicht. Wir schauen und beobachten. Es ist das Praxispersonal, das entscheidet, genau in diesem Punkt möchten wir etwas verändern", meint Hans-Peter Wyss. Qualitätsarbeit als Spiegel der eigenen Praxis, die in den Händen der Praktikerinnen und Praktiker bleiben soll. Das verlangt auch nach Engagement und Eigeninitiative der Ärztinnen, Ärzte und MPAs. Michael Deppeler meint dazu: «Die Definition von Qualität, deren Messung und Kontrolle muss sich auf ärztliches Denken und den Dialog mit den Patienten stützen.»

\section{Die Digitalisierung als neue Aufgabe}

Mit dieser Haltung will die Stiftung in die Zukunft gehen. Schliesslich arbeitet auch die Zeit: Seit etwa 2011, so Hans-Peter Wyss, habe etwa die Digitalisierung der Praxis an Fahrt aufgenommen. Management und Schutz der Daten werden zur Herausforderung. Personalfragen haben mit zunehmender Fluktuation und Grösse der Arztpraxen an Brisanz gewonnen. Und was heisst das für die Teamkommunikation, wenn auf einmal über die Hälfte der Mitarbeitenden in Teilzeit beschäftigt sind? Zu solchen und anderen aktuellen Fragen will die Stiftung - in enger Kooperation mit den Praktikern und Praktikerinnen der ambulanten Medizin - im kommenden Jahr neue Qualitätsprogramme entwickeln.

\footnotetext{
1 Siehe u.a. Bourdieu P. Esquisse d'une théorie de la pratique. Précédée de trois Etudes d'Ethnologie Kabyle. Editions du Seuil; 2000.
}

\section{Nachruf}

Noch während der Redaktion dieser Zeilen, die eigentlich Anlass zur Freude sind, mussten wir von einem der Gründer der EQUAM Stiftung, Dr. med. et lic. oec. Kurt Hess, Abschied nehmen. Er verstarb unerwartet im Alter von 72 Jahren. Er war nicht nur eine prägende Figur für die Stiftung, sondern auch ein treuer Wegbegleiter und Freund für viele. Ein Nachruf wird in einer der nächsten Ausgaben der Ärztezeitung folgen. 